\title{
HÖLDER ESTIMATES FOR THE CAUCHY-RIEMANN EQUATION ON PARAMETERS
}

\author{
SANGhyun Cho
}

AbStract. Let $\left\{\Omega_{\tau}\right\}_{\tau \in I}$ be a family of strictly convex domains in $\mathbb{C}^{n}$. We obtain explicit estimates for the solution of the $\bar{\partial}$-equation on $\Omega \times I$ in Hölder space. We also obtain explicit point-wise derivative estimates for the $\bar{\partial}$-equation both in space and parameter variables.

\section{Introduction}

Let $I \subset \mathbb{R}^{d}$ be a bounded open set containing 0 , and let $\left\{\Omega_{\tau}\right\}_{\tau \in I}$ be a family of smoothly bounded domains in $\mathbb{C}^{n}$ with smooth defining function $\rho_{\tau}$ for $\Omega_{\tau}$ for each $\tau \in I$, and set

$$
\Omega_{I}:=\bigcup_{\tau \in I} \Omega_{\tau} \times\{\tau\}=\Omega \times I .
$$

Then $\Omega_{I}$ is a bounded domain in $\mathbb{R}^{2 n+d}$.

Definition 1.1. $\left\{\Omega_{\tau}\right\}_{\tau \in I}$ is said to be a smooth strongly convex perturbation family of $\Omega_{0}$ if $\Omega_{\tau}$ is strongly convex for each $\tau \in I$ and there is a family of diffeomorphisms $\left\{\Psi_{\tau}\right\}_{\tau \in I}$ such that

(1) $\Psi_{\tau}: \bar{\Omega}_{0} \rightarrow \bar{\Omega}_{\tau}, \Psi_{0}=$ Identity,

(2) $\Psi_{\tau}\left(b \Omega_{0}\right)=b \Omega_{\tau}$ for each $\tau \in I$ and $\Psi_{\tau}$ is smooth on $\tau \in I$ variable.

The solvability of the Cauchy-Riemann equation ( $\bar{\partial}$-equation) and the tangential Cauchy-Riemann equation ( $\bar{\partial}_{b}$-equation) and the estimates of the solutions in various topologies such as in Sobolev, $L^{p}$ and Hölder spaces are key subjects in several complex variables for last several decades $[1,6,7,8,9,10]$.

To study the local behavior of the solutions of $\bar{\partial}$ or $\bar{\partial}_{b}$ equation, we sometimes need to construct a family of strongly pseudoconvex (or strongly convex) domains which are foliated inside of a domain near a boundary point. Let $D \subset \mathbb{C}^{n}$ be a domain and $b D$ is smooth near $z_{0} \in b D$ and the Levi-form of

Received August 27, 2009; Revised November 28, 2009.

2010 Mathematics Subject Classification. 32W05, 32W10.

Key words and phrases. Hölder estimates, integral representation for $\bar{\partial}$, Cauchy-Riemann equation.

Partially supported by KRF-2005-070-C00007 from KRF and by Sogang University. 
$b D$ has $k$ positive eigenvalues at $z_{0}$. In this situation, the author constructed a family of smooth strongly convex family of domains of complex dimension $k$ which are foliated inside of $D$, making a neighborhood of $z_{0}$ in $\bar{D}$, and got estimates of $\bar{\partial}$ and $\bar{\partial}_{b}$ equation in Sobolev spaces near $z_{0} \in b D[3]$.

Let $W$ be a set and $k$ be a nonnegative integer and $0<\alpha \leq 1$. We say $f \in C^{k, \alpha}(W)$ if the norm defined by

$$
|f|_{k, \alpha, W}:=|f|_{k, W}+\left|D^{k} f\right|_{\alpha, W}
$$

is finite. Here $|f|_{k, W}$ denotes the $C^{k}$ norm on $W$ and $\left|D^{k} f\right|_{\alpha, W}$ denotes the sum of the Hölder norm of all derivatives of $f$ of order $k$. We set $C^{k, 0}=C^{k}$, and set $C^{0, \alpha}=C^{\alpha}$, the usual Hölder space, and write $|f|_{0,0}=|f|$. We can extend $|\cdot|_{k, \alpha}$ to the $(p, q)$-forms, denoted by $C_{(p, q)}^{k, \alpha}$, by estimating its components.

In this paper, we prove stability of Hölder estimates for $\bar{\partial}$ and obtain pointwise derivative estimates for $\bar{\partial}$-equation on $\Omega \times I$ for space and parameter variables. Concerning the parameter dependence of the $\bar{\partial}$-equation, one can refer to the most fundamental papers of Hamilton [5].

In the sequel, we let $\left\{\Omega_{\tau}\right\}_{\tau \in I}$ be a smooth strongly convex perturbation family of $\Omega_{0} \subset \subset \mathbb{C}^{n}$ with smooth defining function $\rho_{\tau}$ for $\Omega_{\tau}$ for each $\tau \in I$. We also assume that $|I|$, the diameter of $I$, is sufficiently small so that the estimate (2.4) in Section 2 holds. The following theorem is the stability of the Hölder estimates.

Theorem 1.2. For any $f_{\tau} \in C_{(p, q)}\left(\bar{\Omega}_{\tau}\right), 1 \leq q \leq n$, such that $\bar{\partial} f_{\tau}=0$ in $\Omega_{\tau}$ in the distribution sense, there exists $u_{\tau} \in C_{(p, q-1)}^{1 / 2}\left(\Omega_{\tau}\right)$ such that $\bar{\partial} u_{\tau}=f_{\tau}$ in $\Omega_{\tau}$ and

$$
\left|u_{\tau}\right|_{1 / 2\left(\Omega_{\tau}\right)} \leq C\left|f_{\tau}\right|_{\left(\Omega_{\tau}\right)},
$$

where $C$ is a constant independent of $f_{\tau}$ and $\tau \in I$.

When we study function theories on domains in $\mathbb{C}^{n}$, such as extending $\bar{\partial}_{b^{-}}$ closed forms on $b D$ to $\bar{\partial}$-closed forms on $D$ with Hölder or Sobolev estimates, we sometimes need to get derivative estimates for the solutions of $\bar{\partial}$-equation on $\Omega_{I}$ in space as well as in parameter variables. Let $D_{z}^{l_{1}}$ and $D_{\tau}^{l_{2}}$ be the differential operators of order $l_{1}$ in space variables and of order $l_{2}$ in parameter variables respectively. Also if $f=\sum_{|I|=p,|J|=q} f_{I, J} d z^{I} \wedge d \bar{z}^{J} \in C_{(p, q)}^{k}$, we set

$$
\left|D_{z}^{l} f(z)\right|:=\sum_{|I|=p,|J|=q}\left|D_{z}^{l} f_{I, J}(z)\right|,
$$

where $\left|D_{z}^{l} f_{I, J}(z)\right|=\sum_{|\alpha|=l}\left|D_{z}^{\alpha} f_{I, J}(z)\right|$ and $\alpha$ is a multi-index.

We have the following derivative estimates for the $\bar{\partial}$-equation in Hölder space. As for the estimates in Sobolev space [3], we lose some regularity.

Theorem 1.3. Assume $f_{\tau} \in C_{(0, q)}^{k, \alpha}\left(\bar{\Omega}_{\tau}\right), 1 \leq q \leq n, 0<\alpha<1$, and $\bar{\partial} f_{\tau}=0$ (in distribution sense if $k=0$ ) in $\Omega_{\tau}$. Then there exists $u_{\tau} \in C_{(0, q-1)}^{k+1}\left(\Omega_{\tau}\right)$ 
such that $\bar{\partial} u_{\tau}=f_{\tau}$ in $\Omega_{\tau}$, and for each $0<\alpha^{\prime}<\alpha$ and for each $z_{\tau} \in \Omega_{\tau}$, we have

$$
\begin{aligned}
& \quad\left|D_{z_{\tau}}^{l} u_{\tau}\left(z_{\tau}\right)\right|+\left|D_{\tau}^{l} u_{\tau}\left(z_{\tau}\right)\right| \\
& \leq C_{\alpha, \alpha^{\prime}}\left(\left|\rho_{\tau}\left(z_{\tau}\right)\right|^{-\alpha^{\prime}} \sum_{0 \leq j \leq l-1}\left|D_{z_{\tau}}^{j} f_{\tau}\left(z_{\tau}\right)\right|+\left|f_{\tau}\right|_{l-1, \alpha}\right. \\
& \left.\quad+\left|\rho_{\tau}\left(z_{\tau}\right)\right|^{-l+\frac{\alpha^{\prime}+1}{2}}\left|f_{\tau}\right|_{\alpha}+\left|\rho_{\tau}\left(z_{\tau}\right)\right|^{-l+\frac{1}{2}}\left|f_{\tau}\left(z_{\tau}\right)\right|\right)
\end{aligned}
$$

for $1 \leq l \leq k+1$, where $C_{\alpha, \alpha^{\prime}}$ is a constant independent of $\tau \in I$ and $f_{\tau}$.

Remark 1.4. In Theorem 1.2, we gain $1 / 2$ derivatives. We note that

$$
|\rho(z)|^{-s}|f|_{l, \alpha}
$$

is more or less same as $|f|_{s+l, \alpha}$ in a sense. Therefore we may regard the third term in the right hand side of (1.2) is equivalent to $|f|_{l+\alpha-1 / 2-\alpha^{\prime} / 2}$. Assuming that $0<\alpha \leq 1 / 2$, we therefore gain $1 / 2+\alpha^{\prime} / 2-\alpha$ derivatives which is smaller than $1 / 2$ because $\alpha>\alpha^{\prime}$.

In some cases, $f$ is given in the form $f=\rho^{k-1+\alpha} g$ for some $k \geq 1,0<\alpha<1$ and for some form $g$. In this case the estimate in (1.2) becomes:

Corollary 1.5. Under the same assumptions as in Theorem 1.3, we further assume that $D^{l} f_{\tau}=0$ on $b \Omega_{\tau}$, for each $0 \leq l \leq k-1$, for some $k \geq 1$. Then

$$
\left|D_{z}^{k} u_{\tau}\left(z_{\tau}\right)\right|+\left|D_{\tau}^{k} u_{\tau}\left(z_{\tau}\right)\right| \leq C_{\alpha}\left|f_{\tau}\right|_{k-1, \alpha} .
$$

Remark 1.6. (1) When $k=1$, (1.3) is the derivative estimate of the solution both on space and parameter variables.

(2) Let $D$ be a smoothly bounded domain in $\mathbb{C}^{n}$ with smooth defining function $\rho$, and let $h_{0}$ be a $\bar{\partial}_{b}$-closed form on $b D$. To solve $\bar{\partial}$-closed extension problem on $D$ with Hölder estimates, we first extend $h_{0}$ to $h$ defined on $D$ so that $f:=\bar{\partial} h=\rho^{k-1+\alpha} g$ for some $k \geq 1,0<\alpha<1$ and for some form $g$, depending on the regularity of $h_{0}$.

\section{Integral representation on parameter}

Let $\Omega_{0}$ be a smoothly bounded strongly convex domain in $\mathbb{C}^{n}$ and let $\left\{\Omega_{\tau}\right\}_{\tau \in I}$ be a smooth strongly convex perturbation family of $\Omega_{0}$ with smooth defining function $\rho_{\tau}$ for $\Omega_{\tau}$ for each $\tau \in I$. In this section, we consider the solutions of $\bar{\partial}$-equation in Hölder space in space and parameter variables and prove the stability of the solution in Hölder space as well as pointwise derivative estimates of the solution. In the sequel, we let $A \lesssim B$ mean that there is a constant $C$, independent of parameter $\tau$, such that $A \leq C B$ ( $C$ may be different from line to line).

Definition 2.1. Let $D$ be a domain in $\mathbb{C}^{n}$ with smooth boundary $b D$. A $C^{1}$ function $G(\zeta, z)=\left(g_{1}(\zeta, z), \ldots, g_{n}(\zeta, z)\right)$ is called a Leray map for $D$ if it satisfies $\langle G(\zeta, z), \zeta-z\rangle \neq 0$ for every $(\zeta, z) \in b D \times D$. 
For each $\tau \in I$, consider the map

$$
G_{\tau}^{1}(\zeta, z):=\left(\frac{\partial \rho_{\tau}}{\partial \zeta}\right)=\left(\frac{\partial \rho_{\tau}}{\partial \zeta_{1}}, \ldots, \frac{\partial \rho_{\tau}}{\partial \zeta_{n}}\right),
$$

where $\rho_{\tau}=\rho_{\tau}(\zeta)$ is a boundary defining function for $\Omega_{\tau}$. Then $G_{\tau}^{1}$ is independent of $z$, and it is a Leray map because $\Omega_{\tau}$ is a strongly convex domain for each $\tau \in I$. Using $G_{\tau}^{1}$, we set

$$
\begin{aligned}
& \Phi^{0}=\left(\frac{1}{2 \pi i}\right)^{n} \frac{\langle\bar{\zeta}-\bar{z}, d \zeta\rangle}{|\zeta-z|^{2}} \wedge\left(\frac{\langle d \bar{\zeta}-d \bar{z}, d \zeta\rangle}{|\zeta-z|^{2}}\right)^{n-1}, \\
& \Phi_{\tau}^{1}=\left(\frac{1}{2 \pi i}\right)^{n} \frac{\left\langle G_{\tau}^{1}, d \zeta\right\rangle}{\left\langle G_{\tau}^{1}, \zeta-z\right\rangle} \wedge\left(\frac{\left\langle\bar{\partial}_{\zeta, z} G_{\tau}^{1}, d \zeta\right\rangle}{\left\langle G_{\tau}^{1}, \zeta-z\right\rangle}\right)^{n-1}
\end{aligned}
$$

and

$$
\begin{aligned}
\Phi_{\tau}^{01}= & \left(\frac{1}{2 \pi i}\right)^{n} \frac{\langle\bar{\zeta}-\bar{z}, d \zeta\rangle}{|\zeta-z|^{2}} \wedge \frac{\left\langle G_{\tau}^{1}, d \zeta\right\rangle}{\left\langle G_{\tau}^{1}, \zeta-z\right\rangle} \\
& \wedge \sum_{k_{1}+k_{2}=n-2}\left(\frac{\langle d \bar{\zeta}-d \bar{z}, d \zeta\rangle}{|\zeta-z|^{2}}\right)^{k_{1}} \wedge\left(\frac{\left\langle\bar{\partial}_{\zeta, z} G_{\tau}^{1}, d \zeta\right\rangle}{\left\langle G_{\tau}^{1}, \zeta-z\right\rangle}\right)^{k_{2}},
\end{aligned}
$$

where $\bar{\partial}_{\zeta, z}=\bar{\partial}_{\zeta}+\bar{\partial}_{z}$. We use the notation $\Phi_{q}^{0}, \Phi_{q, \tau}^{1}$ and $\Phi_{q, \tau}^{01}$ to denote the summand of forms with degree $(0, q)$ in $z$ in $\Phi^{0}, \Phi_{\tau}^{1}$ and $\Phi_{\tau}^{01}$ respectively. Note that $\Phi^{0}$ is independent of $\tau$ and $\Phi_{\tau}^{1}$ and $\Phi_{\tau}^{01}$ depend on $G_{\tau}^{1}$ which is the derivatives of $\rho_{\tau}$.

Since $\Omega_{\tau} \subset \mathbb{C}^{n}$ is strongly convex, the denominators of $\Phi_{\tau}^{1}$ and $\Phi_{\tau}^{01}$ are different from zero for $\zeta \in b \Omega_{\tau}$ and $z \in \Omega_{\tau}$, and they depend smoothly on $\tau$. We have the following homotopy formula for $\bar{\partial}$ on convex domains by LerayKoppelman.

Theorem 2.2. For $f_{\tau} \in C_{(0, q)}^{1}\left(\bar{\Omega}_{\tau}\right), 1 \leq q \leq n$, we have

$$
f_{\tau}(z)=\bar{\partial}_{z} T_{\tau}^{q} f_{\tau}(z)+T_{\tau}^{q+1} \bar{\partial} f_{\tau}(z), \quad z \in \Omega_{\tau},
$$

where

$$
T_{\tau}^{q} f_{\tau}(z)=\int_{\Omega_{\tau}} \Phi_{q-1}^{0}(\zeta, z) \wedge f_{\tau}(\zeta)-\int_{b \Omega_{\tau}} \Phi_{q-1, \tau}^{01}(\zeta, z) \wedge f_{\tau}(\zeta)
$$

Note that $\rho_{\tau} \longrightarrow \rho_{0}$ in $C^{\infty}$ topology as $\tau \rightarrow 0$ and there is a family of diffeomorphisms $\Psi_{\tau}: \bar{\Omega}_{0} \rightarrow \bar{\Omega}_{\tau}, \Psi\left(b \Omega_{0}\right)=b \Omega_{\tau}, \Psi_{0}$ is identity and $\Psi_{\tau}$ is smooth on $\tau \in I$ variable. Since $\Omega_{0}$ is strongly convex and $\rho_{\tau}=\left(\Psi_{\tau}^{-1}\right)^{*} \rho_{0}$, there is a uniform constant $c>0$, independent of $\tau \in I$, such that

$$
c|a|^{2} \leq \sum_{i, j=1}^{2 n} \frac{\partial^{2} \rho_{\tau}}{\partial x_{i} \partial x_{j}}(x) a_{i} a_{j}
$$


for all $x \in b \Omega_{\tau}$ and $a \in \mathbb{R}^{2 n}$ provided $|I|$, the diameter of $I$, is sufficiently small. The estimate in (2.4) is essential in the estimates of Lemma 11.2.9 in [2] and we obtain that

Lemma 2.3. There is a constant $c$, independent of $\tau \in I$, such that for any $\zeta \in b \Omega_{\tau}$, and $z \in \bar{\Omega}_{\tau}$,

$$
\operatorname{Re}\left\langle G_{\tau}^{1}, \zeta-z\right\rangle \geq c\left(-\rho_{\tau}(z)+|\zeta-z|^{2}\right) .
$$

Set

$$
h_{\tau}^{1}(\zeta, z)=\left\langle G_{\tau}^{1}, \zeta-z\right\rangle \text { and } h_{\tau}^{0}(\zeta, z)=|\zeta-z|^{2} .
$$

As in the proof of Lemma 11.2.10 in [2], for each fixed $z_{\tau}$ near $b \Omega_{\tau}$, there is a special coordinate system $(t, y)=\left(t^{\prime}, t_{2 n-1}, y\right)$ defined in a neighborhood $U$ of $z_{\tau}$ such that $t_{i}\left(z_{\tau}\right)=0$, and $y=\rho_{\tau}(\zeta), t_{2 n-1}=\operatorname{Im} h_{\tau}^{1}(\zeta, z)$. Then the estimate in (2.5) shows that there exist constants $c_{0}>0$ and $C_{0}$ independent of $\tau \in I$ such that

$$
\begin{aligned}
h_{\tau}^{1}(\zeta, z) & \geq c_{0}\left(\left|\rho_{\tau}(z)\right|+\left|t^{\prime}\right|^{2}+\left|t_{2 n-1}\right|\right), \\
c_{0}\left(\left|\rho_{\tau}(z)\right|+|t|\right) & \leq|\zeta-z| \leq C_{0}\left(\left|\rho_{\tau}(z)\right|+|t|\right) .
\end{aligned}
$$

The estimates in (2.6) are the key ingredients to prove the $\frac{1}{2}$-Hölder estimates for $\bar{\partial}$ on strictly convex domains.

Let $\left\{\Psi_{\tau}\right\}_{\tau \in I}$ be the family of diffeomorphisms defined before (2.4) or in the Definition 1.1. By the generalized change of variables theorem, one obtains that

$$
\int_{\Omega_{\tau}} \omega=\int_{\Omega} \Psi_{\tau}^{*} \omega, \text { and } \int_{b \Omega_{\tau}} \omega^{\prime}=\int_{b \Omega} \Psi_{\tau}^{*} \omega^{\prime}
$$

where $\omega$ and $\omega^{\prime}$ are $2 n$ and $2 n-1$ forms on $\Omega_{\tau}$ and $b \Omega_{\tau}$ respectively. Using the relations in (2.7), we may bring the estimates on $\Omega_{\tau}$ onto $\Omega_{0}$.

For any $\Phi_{\tau} \in C_{(p, q)}^{k}\left(\bar{\Omega}_{\tau}\right)$, set $\Phi_{0}=\Psi_{\tau}^{*} \Phi_{\tau} \in C_{(p, q)}^{k}\left(\bar{\Omega}_{0}\right)$. Since $\left|D_{\tau}^{k} \Psi_{\tau}^{-1}\right| \leq \tilde{C}_{k}$, independent of $\tau \in I$, it follows that

$$
\left|D_{\tau}^{k} \Phi_{\tau}\left(z_{\tau}\right)\right| \leq C_{k} \sum_{l=1}^{k}\left|D_{z}^{l} \Phi_{0}(z)\right|
$$

for an independent constant $C_{k}>0$, where $z_{\tau}=\Psi_{\tau}(z)$.

Note that the estimates in (2.5) and (2.6) are the key ingredients to prove the Hölder estimates of $\bar{\partial}$-equation gaining $\frac{1}{2}$ derivative. Since the estimates in (2.5) and (2.6) are independent of $\tau \in I$, and the solution operator in (2.3) is the Bochner-Martinelli integral, we can pull-back the estimates of the solution of $\bar{\partial}$-equation on $\Omega_{0}$ to the estimates of the solution on $\Omega_{\tau}$ via $(2.7)$ and $(2.8)$ with $k=1$. Then the rest of the proof will be the same as in the proof of Theorem 11.2.11 in [2] proving Theorem 1.2, the stability of the Hölder estimates. 


\section{Derivative estimates}

In this section, we want to get derivative estimates for the solutions of $\bar{\partial}$ equation on $\Omega_{\tau}$ in space as well as in parameter variables. As for the estimates in Sobolev space [3], we lose some regularity when we take derivatives of the solution. For each $z, \zeta \in \Omega_{0}$, we set $z_{\tau}=\Psi_{\tau}(z)$ and $\zeta_{\tau}=\Psi_{\tau}(\zeta)$, and we let $\rho_{\tau}(z)=\rho\left(\Psi_{\tau}(z)\right)$.

Lemma 3.1. Assume $g_{\tau} \in C_{0, q}^{k}\left(\Omega_{\tau}\right)$ and set $g=\Psi_{\tau}^{*} g_{\tau}$. Then

$$
\left|D_{z}^{k} g(z)\right| \lesssim \sum_{l=1}^{k}\left|D_{z_{\tau}}^{l} g_{\tau}\left(z_{\tau}\right)\right| \quad \text { and }\left|D_{z_{\tau}}^{k} g_{\tau}\left(z_{\tau}\right)\right| \lesssim \sum_{l=1}^{k}\left|D_{z}^{l} g(z)\right| .
$$

Proof. Note that $\Psi_{\tau} \rightarrow I_{0}$ as $\tau \rightarrow 0$ in $C^{\infty}(\Omega \times I)$ topology, where $I_{0}$ is the identity map on $\Omega_{0}$. Therefore it follows that $\left|D_{z}^{m}\left[\Psi_{\tau}(z)^{p}\right]\right| \leq C_{k}$ for any integers $m, p \leq k$. Since $D_{z}^{k} g(z)$ is the sum of the terms of the form $D_{z_{\tau}}^{l} g_{\tau}\left(z_{\tau}\right) D_{z}^{m}\left[\Psi_{\tau}(z)^{p}\right]$, where $1 \leq l \leq k$ and $m, p \leq k$, the first estimate in (3.1) holds. Similarly, if we consider the map $\Psi_{\tau}^{-1}$, the second estimate in (3.1) holds.

In the sequel, we let $\beta_{\epsilon}(z)$ be a ball of radius $\epsilon>0$ with center at $z$. We need the following integral estimates for the form $\Phi^{0}$ defined in (2.1). We note that $\Phi^{0}$ is independent of $\tau \in I$.

Lemma 3.2. Let $D$ be a smoothly bounded domain in $\mathbb{C}^{n}$ with smooth defining function $\rho$. For each $z \in D$ and for any $\epsilon>0$ such that $\beta_{\epsilon}(z) \subset D$, we set $D_{\epsilon}=D \backslash \beta_{\epsilon}(z)$. Then for any $\alpha^{\prime}>0$ we have

$$
\int_{b D}\left|\Phi^{0}(\zeta, z)\right||\zeta-z|^{\alpha^{\prime}} \leq C_{\alpha^{\prime}}
$$

and

$$
\left|\int_{D_{\epsilon}} \nabla_{z} \Phi^{0}(\zeta, z)\right| \leq C_{\alpha^{\prime}}|\rho(z)|^{-\alpha^{\prime}}
$$

Proof. Choose a neighborhood $U$ of $z$ where the special local coordinate functions $(t, \rho)=\left(t^{\prime}, t_{2 n-1}, \rho\right)$ are defined where $t=\left(t^{\prime}, t_{2 n-1}\right)$ are tangential coordinates. Using the expression of $\Phi^{0}$ in (2.1), and integrating with respect to $t_{2 n-1}$, and then using polar coordinates $\left|t^{\prime}\right|=r$, we have

$$
\begin{aligned}
\int_{b D \cap U}\left|\Phi^{0}(\zeta, z)\right||\zeta-z|^{\alpha^{\prime}} & \lesssim \int_{b D \cap U}|\zeta-z|^{-2 n+1+\alpha^{\prime}} \\
& \lesssim \int_{|t| \leq A} \frac{d t_{1} d t_{2} \cdots d t_{2 n-1}}{\left(\left|t_{2 n-1}\right|+\left|t^{\prime}\right|\right)\left|t^{\prime}\right|^{2 n-2-\alpha^{\prime}}} \\
& \lesssim \int_{0}^{A} \frac{r^{2 n-3}|\ln r| d r}{r^{2 n-2-\alpha^{\prime}}} \lesssim \tilde{C}_{\alpha^{\prime}}
\end{aligned}
$$


This estimate holds for each coordinate neighborhood and hence it follows that

$$
\int_{b D}\left|\Phi^{0}(\zeta, z)\right||\zeta-z|^{\alpha^{\prime}} \leq C_{\alpha^{\prime}}
$$

This proves (3.2).

To prove (3.3), we use Stokes' theorem and then polar coordinates $|\zeta-z|=r$ :

$$
\begin{aligned}
\left|\int_{D_{\epsilon}} \nabla_{\zeta} \Phi^{0}(\zeta, z)\right| & \leq \int_{|\zeta-z| \geq|\rho(z)|}\left|\nabla_{\zeta} \Phi^{0}(\zeta, z)\right|+\left|\int_{\epsilon \leq|\zeta-z| \leq|\rho(z)|} \nabla_{z} \Phi^{0}(\zeta, z)\right| \\
& \lesssim \int_{|\rho(z)|}^{A} r^{-1} d r+\int_{|\zeta-z|=\epsilon}\left|\Phi^{0}(\zeta, z)\right|+\int_{|\zeta-z|=|\rho(z)|}\left|\Phi^{0}(\zeta, z)\right| \\
& \lesssim|\rho(z)|^{-\alpha^{\prime}},
\end{aligned}
$$

because $|\rho(z)|^{\alpha^{\prime}} \ln |\rho(z)| \lesssim \tilde{C}_{\alpha^{\prime}}$ for each $\alpha^{\prime}>0$, and $\epsilon \lesssim|\rho(z)|$.

To get the derivative estimates of $\bar{\partial}$-equation, we need to get derivative estimates of $\Phi_{\tau}^{01}$. For a convenience, we drop the index $\tau$ in the expressions of $z_{\tau}, \zeta_{\tau}, \rho_{\tau}, h_{\tau}^{1}$ and $\Phi_{\tau}^{01}$ etc. Then we have

$$
\begin{aligned}
& \left|D_{z}^{l} \Phi_{q-1}^{01}(\zeta, z)\right| \\
\lesssim & \sum_{k=1}^{n-1}\left[\sum_{j=1}^{l} \frac{|\zeta-z|}{\left|h^{1}\right|^{n-k+j}|\zeta-z|^{2 k+l-j}}+\sum_{j=1}^{l-1} \frac{1}{\left|h^{1}\right|^{n-k+j}|\zeta-z|^{2 k+l-j-2}}\right] \\
:= & \sum_{k=1}^{n-1}\left[\sum_{j=1}^{l} R_{k j}^{1}(\zeta, z)+\sum_{j=1}^{l-1} R_{k j}^{2}(\zeta, z)\right]:=R_{l}(\zeta, z),
\end{aligned}
$$

where $h^{1}=h^{1}(\zeta, z)$ satisfies the estimate (2.6).

Lemma 3.3. For each real numbers $0<\alpha^{\prime}<\alpha<1$, we have

$$
\begin{aligned}
\int_{b \Omega} R_{l}(\zeta, z)|\zeta-z|^{\alpha} & \leq C_{\alpha, \alpha^{\prime}}|\rho(z)|^{-l+\frac{1+\alpha^{\prime}}{2}}, \quad \text { and } \\
\int_{b \Omega} R_{l}(\zeta, z) & \leq C|\rho(z)|^{-l+1 / 2}
\end{aligned}
$$

Proof. Fix $z \in \Omega$ for a moment and choose a neighborhood $U$ of $z$ where the special local coordinate functions $(t, y)=\left(t^{\prime}, t_{2 n-1}, y\right)$ are defined satisfying the estimates (2.6). Set $\delta=|\rho(z)|$ for a convenience. Let's estimate, for example, the integral containing $R_{k l}^{1}$ term which is the optimal case:

$$
\begin{aligned}
\int_{b \Omega \cap U} R_{k l}^{1}(\zeta, z)|\zeta-z|^{\alpha} & =\int_{b \Omega \cap U} \frac{1}{\left|h^{1}\right|^{n-k+l}|\zeta-z|^{2 k-1-\alpha}} \\
& \leq C \int_{|t| \leq A} \frac{d t_{1} d t_{2} \cdots d t_{2 n-1}}{\left(\delta+\left|t_{2 n-1}\right|+\left|t^{\prime}\right|^{2}\right)^{n-k+l}\left(\delta+\left|t^{\prime}\right|\right)^{2 k-1-\alpha}}
\end{aligned}
$$


for some constants $A$ and $C$ independent of $\tau$, where we have used the estimates (2.6) and the fact that $2 k-1-\alpha \geq 0$. If we integrate the right side of (3.6) with respect to $t_{2 n-1}$ and then use polar coordinates $\left|t^{\prime}\right|=r$, we obtain that

$$
\begin{aligned}
\int_{b \Omega \cap U} R_{k l}^{1}(\zeta, z)|\zeta-z|^{\alpha} & \lesssim \int_{0}^{A} \frac{r^{2 n-3} d r}{\left(\delta+r^{2}\right)^{n-k+l-1}(\delta+r)^{2 k-1-\alpha}} \\
& \lesssim \int_{0}^{A} \frac{r^{2 n-3} d r}{\delta^{l-\frac{1+\alpha^{\prime}}{2}} r^{2 n-2+\alpha^{\prime}-\alpha}} \leq C_{\alpha, \alpha^{\prime}} \delta^{-l+\frac{1+\alpha^{\prime}}{2}}
\end{aligned}
$$

Similarly the other integrals, involving $R_{k j}^{i}$, can be estimated and we get the first part of the estimate in (3.5).

For the second part of the estimate in (3.5), one obtains, as in (3.7), that

$$
\begin{aligned}
\int_{b \Omega \cap U} R_{k l}^{1}(\zeta, z) & \lesssim \int_{0}^{A} \frac{r^{2 n-3} d r}{\left(\delta+r^{2}\right)^{n-k+l-1}(\delta+r)^{2 k-1}} \\
& \lesssim \int_{0}^{A} \frac{d r}{\left(\delta+r^{2}\right) \delta^{l-1}} \leq C \delta^{-l+1 / 2}
\end{aligned}
$$

Now we are ready to prove Theorem 1.3, the pointwise derivative estimates for the solution of $\bar{\partial}$-equation.

Proof of Theorem 1.3. We first assume that $f_{\tau} \in C_{0, q}^{k+1}\left(\bar{\Omega}_{\tau}\right)$. Since $\left|D_{\tau}^{l} \Psi_{\tau}\right| \leq$ $C_{l}$, independent of $\tau \in I$, and by virtue of (2.7) and (2.8), we only need to estimate $\left|D_{z_{\tau}}^{l} u_{\tau}\left(z_{\tau}\right)\right|$. Note that the solution $u_{\tau}$ is given by $u_{\tau}=T_{\tau}^{q} f_{\tau}$ because $\bar{\partial} f_{\tau}=0$ where $T_{\tau}^{q}$ is given in (2.3). Set $f=\Psi_{\tau}^{*} f_{\tau}, u=\left(\Psi_{\tau}\right)^{*} u_{\tau}$, and set $z=\Psi_{\tau}^{-1}\left(z_{\tau}\right)$. As in (2.7), we pull-back our solution to $\Omega_{0}$ and we can write:

$$
\begin{aligned}
u(z)= & \int_{\Omega_{0}} \Phi_{q-1}^{0}\left(\Psi_{\tau}(\zeta), \Psi_{\tau}(z)\right) \wedge f\left(\Psi_{\tau}(\zeta)\right) \\
& -\int_{b \Omega_{0}} \Phi_{q-1, \tau}^{01}\left(\Psi_{\tau}(\zeta), \Psi_{\tau}(z)\right) \wedge f\left(\Psi_{\tau}(\zeta)\right):=u^{0}(z)-u^{1}(z) .
\end{aligned}
$$

In view of Lemma 3.1, it is enough to estimate $\left|D_{z}^{l} u(z)\right|$.

At first, let's estimate the derivatives of $u^{0}(z)$. For a convenience of notation, we set $\Omega_{0}=\Omega, \zeta_{\tau}=\Psi_{\tau}(\zeta)=\zeta$ and $z_{\tau}=\Psi_{\tau}(z)=z$ for each $z, \zeta \in \Omega$. For a moment, we fix $z \in \Omega$ and choose $\epsilon>0$ so that $\beta_{\epsilon}(z) \subset \Omega$, and set $\Omega_{\epsilon}=\Omega \backslash \beta_{\epsilon}(z)$ where $\beta_{\epsilon}(z)$ is the ball of radius $\epsilon>0$ with center at $z$. Then we can write

$$
u^{0}(z)=\int_{\Omega_{\epsilon}} \Phi_{q-1}^{0}(\zeta, z) \wedge f(\zeta)+\int_{\beta_{\epsilon}(z)} \Phi_{q-1}^{0}(\zeta, z) \wedge f(\zeta):=I_{1}^{\epsilon}(z)+I_{2}^{\epsilon}(z) .
$$

Assume that $f=f_{J} d \bar{\zeta}^{J}$, where $J=(1, \ldots, q)$ and set $c_{n}=\frac{(n-1) !}{(2 \pi i)^{n}}$. Using the relation $\frac{\partial}{\partial \zeta_{j}}\left(\frac{1}{|\zeta-z|^{2 n-2}}\right)=-2(n-1) \frac{\bar{\zeta}_{j}-\bar{z}_{j}}{|\zeta-z|^{2 n}}$, and by using Stokes' theorem, 
it follows that

(3.10)

$$
\begin{aligned}
& I_{2}^{\epsilon}(z) \\
= & c_{n} \sum_{j=1}^{q} \frac{(-1)^{j+1}}{n-1} \int_{\beta_{\epsilon}(z)} \frac{\partial}{\partial \zeta_{j}}\left(\frac{1}{|\zeta-z|^{2 n-2}}\right) f_{J}(\zeta) \wedge d \bar{z}^{1 \cdots \hat{j} \cdots q} \\
= & c_{n} \sum_{j=1}^{q} \frac{(-1)^{j}}{n-1}\left(\int_{b \beta_{\epsilon}(z)} \frac{1}{|\zeta-z|^{2 n-2}} f_{J}(\zeta)\left[\hat{\zeta}_{j}\right]+\int_{\beta_{\epsilon}(z)} \frac{1}{|\zeta-z|^{2 n-2}} \frac{\partial f_{J}}{\partial \zeta_{j}}(\zeta)\right) \\
& \wedge d \bar{z}^{1 \cdots \hat{j} \cdots q}
\end{aligned}
$$

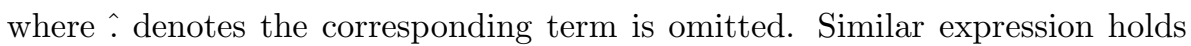
for general $(0, q)$-form $f$. Therefore it follows that

$$
\begin{aligned}
\left|D_{z} I_{2}^{\epsilon}(z)\right| & \lesssim \int_{b \beta_{\epsilon}(z)}\left|D_{z}\left(|\zeta-z|^{-2 n+2}\right)\right||f(\zeta)|+\int_{\beta_{\epsilon}(z)}\left|D_{z}\left(|\zeta-z|^{-2 n+2}\right)\right|\left|\frac{\partial f}{\partial \zeta_{j}}(\zeta)\right| \\
& \lesssim|f|+\mathcal{O}(\epsilon)|f|_{1} .
\end{aligned}
$$

To estimate $\left|D_{z}^{l} I_{2}^{\epsilon}(z)\right|$ for $l \geq 2$, we use the expression of $I_{2}^{\epsilon}(z)$ in (3.10), and use Stokes' theorem inductively together the fact that $D_{z}\left(|\zeta-z|^{2 n-2}\right)=$ $-D_{\zeta}\left(|\zeta-z|^{2 n-2}\right)$. Then one obtains that

$$
\left|D_{z}^{l} I_{2}^{\epsilon}(z)\right| \leq C\left(|f|_{l-1}+\mathcal{O}(\epsilon)|f|_{l}\right)
$$

Now let's estimate $D_{z}^{l} I_{1}^{\epsilon}(z)$. Note that the integrand of $I_{1}^{\epsilon}(z)$ is in $C^{\infty}$ class (in $z$-variable) and hence we can differentiate $I_{1}^{\epsilon}(z)$ under the integral sign. Again we use Stokes' theorem inductively together the fact that $D_{z}(\mid \zeta-$ $\left.\left.z\right|^{2 n-2}\right)=-D_{\zeta}\left(|\zeta-z|^{2 n-2}\right)$ to obtain that

$$
\begin{aligned}
\left|D_{z}^{l} I_{1}^{\epsilon}(z)\right| \lesssim & \left|\int_{\Omega_{\epsilon}} \nabla_{z} \Phi_{q-1}^{0}(\zeta, z) \wedge D_{\zeta}^{l-1} f(\zeta)\right|+\left|\int_{b \Omega} \Phi_{q-1}^{0}(\zeta, z) \wedge D_{\zeta}^{l-1} f(\zeta)\right| \\
& +\left|\int_{b \beta_{\epsilon}(z)} \Phi_{q-1}^{0}(\zeta, z) \wedge D_{\zeta}^{l-1} f(\zeta)\right|:=I_{1 l}^{\epsilon}(z)+I_{2 l}(z)+I_{3 l}^{\epsilon}(z) .
\end{aligned}
$$

Note that

$$
\begin{aligned}
I_{3 l}^{\epsilon}(z) \leq & \left|\int_{b \beta_{\epsilon}(z)} \Phi_{q-1}^{0}(\zeta, z) \wedge\left(D^{l-1} f(\zeta)-D^{l-1} f(z)\right)\right| \\
& +\left|D^{l-1} f(z)\right|\left|\int_{b \beta_{\epsilon}(z)} \Phi_{q-1}^{0}(\zeta, z)\right| \lesssim \mathcal{O}\left(\epsilon^{\alpha}\right)|f|_{l-1, \alpha}+\left|D^{l-1} f(z)\right| .
\end{aligned}
$$


As in (3.14), we write

$$
\begin{aligned}
I_{1 l}^{\epsilon}(z) \lesssim & \int_{\Omega_{\epsilon}}\left|\nabla_{z} \Phi_{q-1}^{0}(\zeta, z)\right|\left|D^{l-1} f(\zeta)-D^{l-1} f(z)\right| \\
& +\left|D^{l-1} f(z)\right|\left|\int_{\Omega_{\epsilon}} \nabla_{z} \Phi_{q-1}^{0}(\zeta, z)\right|:=I_{11 l}^{\epsilon}(z)+I_{12 l}^{\epsilon}(z) .
\end{aligned}
$$

In view of (3.3), we have

$$
I_{12 l}^{\epsilon}(z) \leq C_{\alpha^{\prime}}|\rho(z)|^{-\alpha^{\prime}}\left|D^{l-1} f(z)\right|
$$

for each $\alpha^{\prime}>0$.

Using polar coordinate $|\zeta-z|=r$ and from the fact that $f \in C_{0, q}^{k, \alpha}(\Omega)$, $l-1 \leq k$, it follows, from $(2.1)$, that

$$
\begin{aligned}
I_{11 l}^{\epsilon}(z) & \leq C|f|_{l-1, \alpha(\Omega)} \int_{\Omega}\left|\nabla_{z} \Phi_{q-1}^{0}(\zeta, z)\right||\zeta-z|^{\alpha} \\
& \leq \tilde{C}_{\alpha}|f|_{l-1, \alpha(\Omega)} \int_{0}^{A} r^{\alpha-1} d r \leq C_{\alpha}|f|_{l-1, \alpha(\Omega)}
\end{aligned}
$$

for some $A>|\Omega|$, where $|\Omega|$ denotes the diameter of $\Omega$. Combining (3.16) and (3.17), we obtain that

$$
I_{1 l}^{\epsilon}(z) \leq C_{\alpha^{\prime}, \alpha}\left(|\rho(z)|^{-\alpha^{\prime}}\left|D^{l-1} f(z)\right|+|f|_{l-1, \alpha(\Omega)}\right)
$$

for each $\alpha^{\prime}>0$.

If we use the estimate (3.2), and use the method similar to the estimate (3.15), we obtain that

$$
\begin{aligned}
I_{2 l}^{\epsilon}(z) & \lesssim|f|_{l-1, \alpha(\Omega)}+\left|D^{l-1} f(z)\right||\rho(z)|^{-\alpha^{\prime}} \int_{b \Omega}\left|\Phi_{q-1}^{0}(\zeta, z)\right||\zeta-z|^{\alpha^{\prime}} \\
& \leq \tilde{C}_{\alpha^{\prime}, \alpha}\left(|f|_{l-1, \alpha(\Omega)}+\left|D^{l-1} f(z)\right||\rho(z)|^{-\alpha^{\prime}}\right)
\end{aligned}
$$

because $|\rho(z)| \lesssim|\zeta-z|$ for $\zeta \in b \Omega$. Letting $\epsilon \rightarrow 0$, it follows, from (3.9) and (3.12)-(3.19), that for each $0<\alpha^{\prime}<\alpha<1$, we have

$$
\left|D_{z}^{l} u^{0}(z)\right| \leq C_{\alpha^{\prime}, \alpha}\left(|f|_{l-1, \alpha(\Omega)}+\left|D_{z}^{l-1} f(z)\right||\rho(z)|^{-\alpha^{\prime}}\right)
$$

for $l-1 \leq k$ and for $f \in C_{0, q}^{k+1}(\Omega)$, where $C_{\alpha^{\prime}, \alpha}$ is independent of $\tau \in I$.

By regularization process, we can approximate $f \in C_{0, q}^{k, \alpha}(\Omega)$ by a sequence of $f_{\delta} \in C_{0, q}^{k+1}(\Omega)$ such that $f_{\delta}$ converges to $f$ in $C^{k, \alpha}(\Omega)$ space, and the corresponding solutions $u_{\delta}^{0}$ converges to $u^{0}$ in $C^{k+1}(\Omega)$ space as $\delta$ converges to zero. Thus (3.20) holds for $f \in C^{k, \alpha}(\Omega)$. In view of Lemma 3.1, we see that $\left|D_{z_{\tau}}^{l} u_{\tau}^{0}\left(z_{\tau}\right)\right|$ is bounded by the first two terms in the right hand side of (1.2).

Next we estimate $\left|D^{l} u^{1}(z)\right|$. For a convenience, we also drop the index $\tau$ in the expressions of $z_{\tau}, \zeta_{\tau}, f_{\tau}$ and $\rho_{\tau}$ etc. Choose a neighborhood $U$ of $z$ where the special local coordinate functions $(t, y)=\left(t^{\prime}, t_{2 n-1}, y\right)$ are defined satisfying 
the estimates (2.6). Since $u^{1}$ is a smooth function, we can differentiate under the integral sign. Using (2.2), (2.6), (3.4) and (3.5), one obtains that

$$
\begin{aligned}
& \int_{b \Omega \cap U}\left|D_{z}^{l} \Phi_{q-1}^{01}(\zeta, z) \wedge f(\zeta)\right| \\
\leq & \int_{b \Omega \cap U}\left|D_{z}^{l} \Phi_{q-1}^{01}(\zeta, z)\right||f(\zeta)-f(z)|+|f(z)| \int_{b \Omega \cap U}\left|D_{z}^{l} \Phi_{q-1}^{01}(\zeta, z)\right| \\
\lesssim & |f|_{\alpha} \int_{b \Omega \cap U} R_{l}(\zeta, z)|\zeta-z|^{\alpha}+|f(z)| \int_{b \Omega \cap U} R_{l}(\zeta, z) \\
\lesssim & |\rho(z)|^{-l+\frac{1+\alpha^{\prime}}{2}}|f|_{\alpha}+|\rho(z)|^{-l+1 / 2}|f(z)|
\end{aligned}
$$

and hence it follows that

$$
\left|D^{l} u^{1}(z)\right| \leq C_{\alpha, \alpha^{\prime}}\left(|f|_{\alpha}|\rho(z)|^{-l+1 / 2+\alpha^{\prime} / 2}+|f(z)||\rho(z)|^{-l+1 / 2}\right) .
$$

If we combine (3.22) and Lemma 3.1, we see that (1.2) holds proving Theorem 1.3.

Lemma 3.4. For any real number $\alpha>0$, suppose $f \in C^{\alpha}(D)$ and $f=0$ on $b D$. Then

$$
\sup _{z \in D}|\rho(z)|^{-\alpha}|f(z)| \leq C|f|_{\alpha(D)} .
$$

Proof. For each fixed $z \in D$, let $\zeta_{z}$ be the projection of $z$ onto $b D$. Then it follows that $\left|z-\zeta_{z}\right| \approx|\rho(z)|$. Since $f=0$ on $b D$, we have

$$
|\rho(z)|^{-\alpha}|f(z)|=|\rho(z)|^{-\alpha}\left|f(z)-f\left(\zeta_{z}\right)\right| \leq \tilde{C}_{\alpha}|\rho(z)|^{-\alpha}\left|z-\zeta_{z}\right|^{\alpha}|f|_{\alpha} \leq C_{\alpha}|f|_{\alpha} .
$$

Proof of Corollary 1.5. If we write $u=u^{0}+u^{1}$ as in (3.8), it follows that $u^{1}=0$ because $f_{\tau}=0$ on $b \Omega_{\tau}$. By Theorem 1.3, Lemma 3.1 and Lemma 3.4, and from the given condition that $D^{l} f_{\tau}=0$ on $b \Omega_{\tau}$ for $0 \leq l \leq k-1$, it follows that

$$
\left|D^{l} f(z)\right||\rho(z)|^{-\alpha} \leq C_{\alpha}|f|_{l, \alpha}
$$

for $0 \leq l \leq k-1$. If we combine (3.1), (3.20) and (3.23), we see that (1.3) holds and this proves Corollary 1.5.

\section{References}

[1] H. P. Boas and M.-C. Shaw, Sobolev estimates for the Lewy operator on weakly pseudoconvex boundaries, Math. Ann. 274 (1986), no. 2, 221-231.

[2] S.-C. Chen and M.-C. Shaw, Partial Differential Equations in Several Complex Variables, AMS/IP Studies in Advanced Mathematics, 19. American Mathematical Society, Providence, RI; International Press, Boston, MA, 2001.

[3] S. Cho, Sobolev estimates for the local extension of tangential CR-closed forms on real hypersurfaces in $\mathbb{C}^{n}$, Preprint.

[4] S. Cho and J. Choi, Explicit Sobolev estimates for the Cauchy-Riemann equation on parameters, Bull. Korean Math. Soc. 45 (2008), no. 2, 321-338. 
[5] R. S. Hamilton, Deformation of complex structures on manifolds with boundary I, II, J. Differential Geometry 12 (1977), no. 1, 1-45; J. Differential Geom. 14 (1979), no. 3, 409-473.

[6] J. J. Kohn and H. Rossi, On the extension of holomorphic functions from the boundary of a complex manifold, Ann. of Math. (2) 81 (1965), 451-472.

[7] M. Kuranishi, Strongly pseudoconvex CR structures over small balls. I, An a priori estimate. Ann. of Math. (2) 115 (1982), no. 3, 451-500.

[8] M.-C. Shaw, $L^{2}$-estimates and existence theorems for the tangential Cauchy-Riemann complex, Invent. Math. 82 (1985), no. 1, 133-150.

[9] $L^{p}$ estimates for local solutions of $\bar{\partial}_{\mathrm{b}}$ on strongly pseudo-convex CR manifolds, Math. Ann. 288 (1990), no. 1, 35-62.

[10] _ Local existence theorems with estimates for $\bar{\partial}_{b}$ on weakly pseudo-convex $C R$ manifolds, Math. Ann. 294 (1992), no. 4, 677-700.

Department of Mathematics

SOGANG UNIVERSITY

SEOUl 121-742, Korea

E-mail address: shcho@sogang.ac.kr 